\title{
ÉTUDE PRÉLIMAIRE DES FACTEURS FAVORISANT LA CRÉATION DES SOCIÉTÉS POLYGYNES D'APIS MELLIFICA VAR. LIGUSTICA
}

\author{
T. LENSKY et R. DARCHEN \\ Iraculte' d'Agriculture de Rehovot, Israël; \\ Station de Recherches sur l'Abeille et les Insectes sociaux, Bures-sur-Yvette (Seine-el-Oise)
}

\section{SOMMAIRE}

I $^{0}$ A l'occasion de la recherche des facteurs favorisant la création de ruches polygynes, les auteurs ont présenté une technique de formation de sociétés polygynes d'Apis mellifica, var. ligustica. Ils ont montré qu'il fallait obtenir progressivement une odeur commune de tous les individus rechercher un état physiologique semblable des reines, réunir lentement des colonies à reine unique.

$2^{\circ}$ ) Ils ont en outre signalé que les reines cohabitent assez facilement après l'ablation du dard et que les ouvrières surtout sont responsables de la plupart des difficultés rencontrées dans la création des ruches polygynes.

$3^{\circ}$ ) Ils ont observé des phénomènes particuliers montrant que l'action de la phéromone (Queen substance) reste un phénomène limité et que, même dans le cas du blocage de la construction des cellules royales, elle n'est pas seule à intervenir.

$\left.4^{\prime \prime}\right)$ Ils ont enfin indiqué que, dans les ruches polygynes, les ouvrières construisent aussi des cellules inâles.

\section{IN'TRODUCTION}

Les colonies d'A pis mellifica sont reconnues par tous comme des sociétés monogygynes car les reines s'y livrent des combats mortels jusqu'à ce qu'une seule d'entre elles subsiste. Cependant, on a plusieurs fois signalé que les colonies d'Abeilles pouvaient contenir temporairement plusieurs reines (Dool,ITTI,E, KELSAI,I, DAVIS, MATHIS). Dès lors, n'était-il pas possible de trouver des moyens de prolonger anormalement la vie de ces colonies naturellement polygynes ou mieux d'en créer un certain nombre pour connaitre les conséquences de cette cohabitation?

\section{MATÉRIEI, ET MÉTHODE}

Les reines et les ouvrières utilisées appartenaient toutes à la race italinene (Apis mellifica, var. ligustica). L'âge des reines dans les différentes expériences variait entre 3 semaines et 2 ans.

Comme l'agressivité est de règle entre les reines d'Apis mellifica, nous avons décidé de sectionner les dards de toutes celles que nous avons utilisées (BOTTCiter, Darchen, LenSKY).

Les expériences de ce mémoire ont été effectuées en 1961 et 1962 à la Faculté d'Agriculture de Rehovot (Israël). 


\section{ANALYSE DES RÉSULTATS}

Pour faciliter la compréhension des résultats, nous les groupons sous 3 têtes de chapitre.

\section{A) IMPORTANCE, DE L'ÉTAT PHYSIOLOGIQUE DES REINES POUR LA CRÉATION D'UNE POLYGYNE}

Io) Quatorze reines isolées dans des cagettes individuelles ont été introduites deux par deux dans 7 colonies très faibles. I)ans un cas seulement il y a eu acceptation simultanée de deux reines, soit une réussite de I $_{5} \mathrm{p}$. Ioo environ. D'après nos observations, ce sont les ouvrières qui éliminent les reines et non pas les reines qui s'entretuent.

$\left.2^{\circ}\right)$ Deux jeunes reines de 3 semaines ont été introduites au moyen de cagettes grillagées chacune respectivement dans un corps de ruche LANGSTROTH. Les deux corps de ruche, étant superposés, contenaient des abeilles d'une même colonie mais n'étaient séparés par aucune grille à reine ni par aucun papier. Les deux reines ne se sont pas mises à pondre en même temps. Celle qui a pondu la première a été immédiatement acceptée et l'autre a été " emballée " par les ouvrières. Nous avons répété 4 fois cette expérience et les résultats furent les mêmes. Nous en concluons que l'état physiologique de la reine, ou mieux son état de ponte, est probablement responsable de son acceptation ou de son élimination.

Nous avons ensuite cherché tune technique de réunion des colonies d'abeilles favorisant la cohabitation de reines ayant le même état physiologique.

$\left.3^{\circ}\right)$ Deux populations de jeunes Abeilles pourvues de trois cadres de couvain, de miel et de pollen, ont été superposées et séparées par une grille à reine et du papier-filtre percé de petits trous. Chaque colonie a reçu une reine présentée dans une cagette grillagée. Dès sa libération, chacune des reines s'est mise à pondre dans son corps de ruche. Après quelques jours, nous n'avons supprimé que la grille de séparation puisque le papier-filtre avait été éliminé lentement par les ouvrières des 2 colonies. Les trois cadres du corps supérieur ont été descendus à côté des trois cadres inférieurs. Cette expérience a été répétée 5 fois. Dans tous les cas, une des reines a été éliminée. L'échec a donc encore été total et il a fallu rechercher un nouveau perfectionnement à la technique puisque l'obtention d'une odeur commune pour les 2 colonies et d'un état physiologique commun aux deux reines est insuffisant pour réussir la création d'une société polygyne d'abeilles.

\section{B) IMPORTANCE DE LA TECHNIQUE DE RÉUNION DES DEUX COLONIES}

Nous avons répété 1'expérience $A$. 3, mais après le retrait de la grille cle séparation, nous avons laissé les abeilles et les reines se mêler lentement sans déplacer les cadres. Après quelques jours, les reines se trouvaient très souvent l'une près de l'autre, pondant normalement. Chacune possédait une cour mouvante d'environ Io sujets. Les résultats de ces expériences ont été bien meilleurs puisque nous avons obtenu une réussite d'environ $65 \mathrm{p}$. Ioo (4 réunions sur 6 ont réussi). 


\section{C) OBSERVATION DE QUELQUES PHÉNOMÈNES DÉCOULANT DE IA COHABITATION DES REINES}

\section{a) Le remplacement rapide des reines acceptées.}

Dans plus de 80 p. Ioo de ruches polygynes, les ouvrières ont construit des cellules royales pour remplacer leurs reines. Ce phénomène est apparu dans des sociétés faibles ou fortes, avec des reines jeunes ou vieilles, dans une période de temps de quelques semaines ou de quelques mois. Des cellules royales ont été aussi construites dans les 2 cas particuliers suivants :

$\left.I^{\circ}\right)$ dans une colonie très forte (4 corps de ruche) possédant 3 vieilles reines ;

$2^{\circ}$ ) dans une colonie très faible ( 3 cadres de couvain) possédant 3 reines de 3 semaines. Dans tous les exemples cités ici, toutes les reines pondaient régulièrement et étaient entourées d'une cour d'environ ro sujets. Comme nous l'avons déjà écrit, ces observations sont totalement contraires à celles de Kovrun.

b) Au printemps, les abeilles d'une ruche à 3 reines ont construit des cellules de mâles.

Notons que ces résultats sont encore différents de ceux de Kovtun.

c) L'hivernage d'une seule colonie, que nous avions conservée à dessein, a été très bon.

Cette colonie s'est développée rapidement pendant une année; les reines, établies sur quelques cadres au mois d'août avec une très faible poptulation, ont pondu énormément pendant tout l'hiver; la population s'étendait sur 4 corps de ruche a11 printemps; 1 a récolte dépassa de 80 p. Ioo celle des ruches à reine unique. Ce résultat n'a qu'une valeur indicative car il est isolé.

$\mathrm{I}^{\text {re }}$ question.

\section{DISCUSSION}

Pourquoi la cohabitation des reines dépend-elle de leur état physiologique? Lorsque nous réunissons lentement deux colonies contenant chacune une reine, en très bon état, entourée d'une cour normale, nous avons découvert que la réussite de la cohabitation dépend de la ponte des deux reines. Si l'une des deux reines ne pond pas en même temps que 1'autre, elle est supprimée par les abeilles en dépit de toutes les précautions que l'on peut prendre par ailleurs.

SNELGROve a déjà remarqué que les reines vierges sont plus difficilement acceptées par les ouvrières et qu'au contraire les reines fécondes le sont plus facilement. Il ajoute cette remarque, importante pour notre sujet, que " les reines fécondes qui ont cessé de pondre pour une raison quelconque sont plus difficilement acceptées que celles qui pondent immédiatement ". Il explique ce phénomène d'une façon étonnante et qui rappelle les récents travaux de GARY et de BUTLER sur l'attractivité des reines ou sur leur capacité de bloquer la formation des cellules royales : "Elles deviennent, en ne pondant pas, semblables en apparence et en attitude aux reines vierges. Elles ne sollicitent pas la nourriture aussi fortement que les reines pondeuses. Enfin, il est probable que leur odeur caractéristique change quand elles 
cessent de pondre. " A cette occasion, il faut rappeler les travaux de PaIN sur 1'attractivité des reines vierges ou fécondes qui est très différente selon les individus, leur état de fécondité ou bien selon la population des abeilles qui les entourent.

$2^{\text {eme }}$ question.

Pourquoi la méthode de réunion lente réussit-elle? Rappelons à ce sujet que dans les expériences rapportées plus haut les deux colonies à une reine établies sur trois cadres ne sont plus séparées que par une feuille de papier ou par une grille à reine. Il semble donc qu'elles ne forment plus qu'une colonie par le mélange des ouvrières et par cette odeur communiquée par le couvain, le miel et le pollen dont RIBBANDS (I953) a montré l'importance. En fait, l'unité d'une ruche dépend aussi, nous semblet-il, d'autres facteurs tout aussi importants et que nous essayons d'analyser. Au début de la suppression de la grille de séparation, nous nous trouvons en face de deux colonies qui ne sont encore unies que superficiellement par une odeur commune et par un certain mélange des butineuses. Il doit donc exister autour de chaque reine une zone d'influence chimique caractéristique de chacune d'entre elles, faite d'un mélange complexe différemment dosé de substances semblables. Il y aurait une similitude de la qualité des substances mais non de leurs proportions. Seules les butineuses qui vont d'une colonie à l'autre auraient acquis une valeur "moyenne". Un mélange trop rapide des populations aurait pour effet d'empêcher l'égalisation progressive des substances communes issues des deux reines. Rappelons à ce propos que les travaux récents de GARY (I962) et de BUTLER (I96I) vont en ce sens puisque même ce dernier admet maintenant l'existence d'un facteur attractif des reines différent de la phérormone royale (Queen substance). PAIN (I959) l'avait signalé deptris longtemps à la suite de nombreuses expériences.

$3^{\mathrm{e}}$ question.

Pourquoi les Abeilles construisent-elles de nouvelles cellules royales même en présence de plusieurs reines fécondes, jeunes ou vieilles? Cette observation est générale en Israël avec des reines d'A pis mellifica, var. ligustica. Le phénomène survient généralement en dehors de la période d'essaimage. Ce n'est d'ailleurs pas une caractéristique des ruches à plusieurs reines puisqu'un grand nombre de colonies changent de reine chaque année.

On attribue généralement la supersédure à un appauvrissement de la colonie en phérormone, soit par suite d'une défectuosité de la reine, soit par suite d'une mauvaise répartition de la substance entre les ouvrières. Dans le cas qui nous occupe, cette hypothèse ne peut pas être retenue puisque nos colonies polygynes ont des reines jeunes qui pondent normalement et qui sont entourées d'une cour abondante et puisque le phénomène se produit également dans les colonies monogynes pourvues d'une jeune reine. Pour la même raison on ne peut pas non plus invoquer l'hypothèse d'un excès de phérormone qui a été émise par GARY (I962). On sait que cet auteur admet qu'un excès de phérormone a la même action qu'un défaut sur la construction des cellules royales.

I a superséđure généralisée que nous avons constatée en Israël et qui n'intéresse pas que nos ruches polygynes pourrait finalement dépendre d'un facteur purement écologique encore indéterminé ou bien être en rapport avec un caractère particulier à la race d'abeille utilisée. 


\section{REMERCIEMENTS}

Les auteurs remercient respectivement l'Université hébraïque de Jérusalem, la Faculté d'Agriculture de Rehovot, section d'Entomologie, pour l'aide financière et amicale apportée dans ces recherches et enfin la ferme collective de Yad Modehai qui a bien voulu mettre à notre disposition plusieurs colonies et plusieurs reines d'Abeilles.

Reçu pour publication en novembre 1962

\section{SUMMARY}

A PRELIMINARY STUDY OF THE FACTORS PROMOTING THE FORMATION of polygynous societies of Apis mellifica, var. ligustica

I - Technics to create polygynous societies of bees, Apis mellifica, var. ligustica, are set forth. It is pointed out that the best ones have to take care of the scent, of the same physiological state of the queens, of a slow assembling of the one queen societies.

2 - After operations of their stings, the queens peacefully live together but the workers often are accountable of many checks.

3 - Strange facts suggest the queen substance is not the panacea throwing light upon almost all the vital phenomena of a colony, even the cessation of the building of the queen cells.

4 - The workers of the multiqueen societies also build male cells.

\section{RÉFÉRENCES BIBLIOGRAPHIQUES}

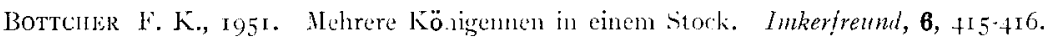

BUTIER C. ( $i$, I950. The simiticance of queen substance in swarning and supersedure in honey Jiee (Apis mellifica) colonies. Proc. Rov. ent. London, 35, 129-132; $196 \mathrm{I}$. The scent of queen honeybees (Apis mellifirt L.) that causs purtial inhibition of (quen rearing. J. Ins. Physiol., 7, $258-264$.

DaRcies R., ig6o. L'ablation du dard des reines et des ouvrières d'Apis mellifica. C. R. Acal. Sci., $250,93 t^{-0} 36$.

DaRchex R., LENSKY J., Igh3. Le probleme des societé polygynes d'Apis mellifuca. Insectes socianx (à paraitre).

Datis J. L., 1908. Queen killed by a rival queen. Glern. Bee Cult., 36, 1259 .

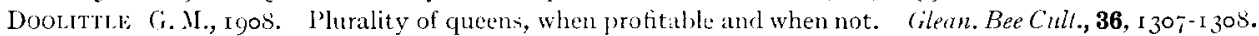

GARI Y. E., 1962. Chemical mating attractunts in the quen honey Bee. Science, 136, 223-224.

Kelsat. A, i940. A multiqueen colony of bees. Amer. Bee J., 80, 170.

Lensky J., DARCHEN R., ig62. Quelques observations sur la construction des cellules royales en présence de reines d'Abeilles (Apis mollifica, var. ligustica). C. R. Lata. Sci. (en parution).

Kovtex I. X., 1950. Colonies à plusicurs reines. Pchelorodsto, 27, i 2.

Matuis M., I95r. Polygynie temporaire mais constante et naturelle chez Apis mellifica, var. punica en Tunisie. Absence de combats mortels entre les reines. (ia. Apic., 53, 230-231.

Pain J., 1959. Etude de l'apparition de l'attractivité chez les reines vierges d'Abeille. C. R. Acal. Sci., 248, $3211-3212$.

Pain igúr. Sur la phérormone d'abeilles et ses effets physiologiques. Ann. Abeille, 4, 73-152.

Ribbixins C. R., 195.3. The behaviour and social life of Iloncybees. 350 p., BRA, London.

SNelGrove L. E., r9łs. The introduction of (queen Bees. 205 p., Snelgrove I, Bleadon. 